\title{
Construção e validação de tecnologia educacional para promoção do aleitamento materno no período neonatala
}

\author{
Construction and validation of educational technology to promote breastfeeding in the neonatal period \\ Construcción y validación de tecnología educativa para promover la lactancia materna en el período \\ neonatal
}

\author{
Mariana Mesquita Silva ${ }^{1}$ (1) \\ Jardeliny Corrêa da Penha ${ }^{1}$ (B) \\ Izabel Cristina Falcão Juvenal Barbosa ${ }^{1}$ (1) \\ Cristianne Teixeira Carneiro ${ }^{2}$ (B) \\ José Wicto Pereira Borges ${ }^{3}$ (D) \\ Maria Augusta Rocha Bezerra ${ }^{1}$ (D)
}

1. Universidade Federal do Piauí, Curso de Graduação em Enfermagem. Floriano, PI, Brasil.

2. Universidade Federal do Piauí, Curso

Técnico de Enfermagem, Floriano, PI, Brasil.

3. Universidade Federal do Piauí,

Departamento de Enfermagem. Teresina, PI, Brasil.
Autor Correspondente:

Maria Augusta Rocha Bezerra.

E-mail:mariaaugusta@ufpi.edu.br

Recebido em 26/06/2020.

Aprovado em 16/09/2020.

DOI:https://doi.org/10.1590/2177-9465-EAN-2020-0235

\section{Resumo}

Objetivo: Este estudo teve como objetivos construir e validar um pacote de mensagens de texto e figuras para promoção do aleitamento materno no período neonatal. Métodos: tratou-se de um estudo metodológico, realizado em quatro etapas: revisão integrativa da literatura, construção de pacote de mensagens e figuras, análise de legibilidade e validação de conteúdo e aparência. Para validação, participaram 11 juízes com expertise em aleitamento materno. $O$ critério para validação foi concordância superior a 80\%, analisada por meio dos índices de validação de conteúdo e aparência. Resultados: a versão final do pacote de mensagens e figuras apresentou índice de validade de conteúdo de 0,86, índice de validade de aparência de 0,85 e validade convergente com correlação de 0,73 entre o conteúdo e a aparência $(p<0,01)$. Conclusões e implicações para a prática: o pacote de mensagens de texto e figuras foi considerado válido em conteúdo, aparência e convergência para promoção do aleitamento materno, no período neonatal, constituindo tecnologia educacional adequada para ser compartilhada via mensagens telefônicas, com intuito de se aproximar das nutrizes, ajudando-as a reagir frente aos problemas comuns no período neonatal.

Palavras-chave: Tecnologia da Informação; Aleitamento Materno; Tecnologia Educacional; Estudo de Validação, Educação em Saúde.

\section{Abstract}

Objective: This study aimed to build and validate a text messages and pictures package to promote breastfeeding in the neonatal period. Methods: It was a methodological study carried out in four stages: integrative literature review, construction of message and picture package, readability analysis and content and appearance validation. For validation, 11 judges with experience in breastfeeding participated. The criterion for validation was agreement of more than $80 \%$, analysed by means of content and appearance validation indexes. Results: the final version of the message and picture package presented a content validity index of 0.86 , appearance validity index of 0.85 and a convergent correlation validity of 0.73 between content and appearance $(p<0.01)$. Conclusions and implications for practice: the package of text messages and figures was considered valid in content, appearance and convergence for the promotion of breastfeeding in the neonatal period, constituting appropriate educational technology suitable to be shared via phone messages, in order to approach the nursing mothers, helping them to react to common problems in the neonatal period.

Keywords: Information Technology; Breast Feeding; Educational Technology; Validation Study; Health Education.

\section{Resumen}

Objetivo: Este estudio tuvo como objetivos construir y validar un paquete de mensajes de texto e imágenes para promover la lactancia materna en el período neonatal. Métodos: fue un estudio estudio metodológico, llevado a cabo en cuatro etapas: revisión integradora de la literatura, construcción de un paquete de mensajes e imágenes, análisis de legibilidad y validación de contenido y la apariencia. El criterio de validación fue un acuerdo superior al $80 \%$, analizado por medio de los índices de validación de contenido y apariencia. Resultados: la versión final del paquete de mensajes y grabados presentó un índice de validez de contenido de 0,86 , un índice de validez de apariencia de 0,85 y una validez de correlación convergente de 0,73 entre contenido y apariencia $(p<0,01)$. Conclusiones e implicaciones para la práctica: el paquete de mensajes de texto y imágenes se consideró válido para el contenido, la apariencia y la convergencia para la promoción de la lactancia materna en el período neonatal, constituyendo una tecnología educativa adecuada para ser compartida a través de mensajes telefónicos, con el fin de acercarse a las madres lactantes, ayudándolas a reaccionar ante problemas comunes en el período neonatal.

Palabras clave: Tecnología de la Información; Lactancia Materna; Tecnología Educacional; Estudio de Validación, Educación en Salud. 


\section{INTRODUÇÃO}

As Tecnologias de Informação e Comunicação (TIC) constituem uma plataforma econômica e permitem acesso instantâneo e comunicação direta para transferência mais rápida de informações. ${ }^{1} \mathrm{Na}$ última década, o quantitativo de usuários dessas tecnologias aumentou significativamente, o que oportunizou o emprego destas entre profissionais de saúde e pacientes nos processos de comunicação em saúde. ${ }^{2}$

Dentre as TIC, destacam-se os telefones celulares, em especial, os smartphones, cuja propagação tem contribuído para o aumento do número de aplicativos disponíveis para download ${ }^{3}$ que, em virtude da facilidade de acesso aos dados, praticidade, disponibilidade e interatividade, têm encontrado diversas funções na área da saúde. ${ }^{4}$ Neste contexto, o aplicativo de mensagens WhatsApp $®$ Messenger emerge como ferramenta útil de assistência à saúde e canal potencial para esclarecer dúvidas, transmitir informações, orientações e promover a adesão ao tratamento. ${ }^{5}$ Configura-se, pois, como tecnologia rápida, confiável e que pode transmitir volumes de dados, com o potencial de revolucionar a comunicação e a tomada de decisões no setor da saúde. ${ }^{6}$

O escopo amplo e a flexibilidade do WhatsApp $®$ Messenger, ajustando-se às necessidades em saúde de cada contexto social, proporcionam soluções inovadoras de prestação de serviços de saúde. O uso inclui fornecer informações em saúde de interesse público, esclarecimento de dúvidas dos usuários, além do envio de tecnologias educacionais. ${ }^{7}$ Assim, considera-se a tecnologia educacional um dispositivo facilitador para promoção da saúde e um recurso cuidativo-educativo. ${ }^{8}$

Entre as temáticas possíveis de serem abordadas na conjuntura dos cuidados em saúde, a partir da utilização do WhatsApp® Messenger e aplicabilidade das tecnologias educacionais, está o Aleitamento Materno (AM). Embora não tenham sido identificados estudos de intervenção individual que empregaram essa tecnologia específica, outras pesquisas indicaram benefícios do enfoque do AM, através de mídias sociais, como discussões para troca de experiências em grupos on-line, ${ }^{9}$ Instagram, ${ }^{10}$ Facebook ${ }^{4}$ e outros aplicativos. ${ }^{11}$

O desenvolvimento e a utilização de tais tecnologias pode implicar sucesso do AM. Do ponto de vista assistencial, o uso de tecnologias da informação na atenção básica, desde o pré-natal até o puerpério, sinaliza mudanças significativas que refletem diretamente nas taxas de AM. No entanto, apesar de iniciativas expressivas terem sido desenvolvidas nos últimos anos, muito ainda precisa ser feito, na perspectiva de elevar os índices de aleitamento exclusivo e complementar, em tempo oportuno, e inibir o desmame precoce. ${ }^{12}$

Mundialmente, as prevalências mais altas de AM estão situadas na África Subsaariana, no Sul da Ásia e em partes da América Latina. Em países de alta renda, a prevalência é inferior a $20,0 \%$, observando-se diferenças significantes, como Reino Unido (<1,0\%), Suécia (16,0\%), Estados Unidos da América $(27,0 \%)$ e Noruega (35,0\%). Contudo, a iniciação precoce do $\mathrm{AM}$, logo após o nascimento, foi baixa em todos os países, assim como de Aleitamento Materno Exclusivo (AME). Em pesquisa recente, identificou-se que entre crianças menores de seis meses, em países de baixa e média renda, 36,3 milhões $(63,0 \%)$ não recebiam AME, no momento da investigação. ${ }^{13}$

Soma-se a isso o fato de que, atualmente, devido à pandemia do novo coronavírus (em inglês coronavirus disease - COVID-19), o distanciamento social passou a ser a medida mais eficaz na redução da disseminação do vírus. Entretanto, essa medida pode dificultar a implementação do suporte necessário empregado pelas equipes de saúde para o estabelecimento e a manutenção do AM. Diante desse cenário, as mídias sociais, em especial, aplicativos como o Whats $A p p \AA$ Messenger podem viabilizar a continuidade da assistência à saúde que transcende o contato físico e possibilitar a promoção do AM no período neonatal.

Sobre isso, estabeleceu-se, dentre as recomendações do sistema de saúde para responder às novas demandas relacionadas à COVID-19, a necessidade de considerar modelos alternativos de atendimento, como prestação de serviços em ambientes e/ou plataformas diferentes (por telefone ou pela Web, por exemplo).$^{14}$

Com base no exposto e em estudos recentes sobre a utilização de aplicativos para a educação em saúde com pacientes, ${ }^{15}$ destaca-se que o uso da tecnologia de smartphones na prática clínica é um tópico de pesquisa crescente. ${ }^{16}$ Por isso, são necessárias pesquisas para explorar como as TIC podem ser aproveitadas para fornecer suporte abrangente ao AM. ${ }^{17,18}$ Deste modo, objetivou-se construir e validar Pacote de Mensagens de Texto e Figuras (PMTF) para promoção do aleitamento materno, no período neonatal.

\section{MÉTODO}

Estudo metodológico, de abordagem quantitativa, desenvolvido de agosto de 2018 a novembro de 2019, em quatro etapas: revisão integrativa da literatura, construção de pacote de mensagens e figuras, análise de legibilidade e validação de conteúdo e aparência.

Quanto à revisão integrativa da literatura (RIL), realizada de janeiro de 2001 a julho de 2019, utilizou-se do National Library of Medicine (PUBMED), do Index to Nursing and Allied Health Literature (CINAHL), da Biblioteca Virtual em Saúde (BVS) Literatura Latino-americana e do Caribe em Ciências da Saúde (LILACS), do WEB of SCIENCE e SCOPUS. Empregaram-se os termos "desmame precoce", "fatores de risco" e "período neonatal" como descritores de pesquisa para selecionar os artigos adequados aos seguintes critérios de inclusão estabelecidos: estudos primários que abordassem os fatores que levam as mães a realizarem o desmame precoce no período neonatal e publicados em inglês, português ou espanhol. Identificaram-se 1.673 artigos potencialmente relevantes para a revisão, destes, 73 foram selecionados para leitura na íntegra, permanecendo 12 artigos na amostra final.

Quanto à construção do PMTF, a seleção do conteúdo foi realizada mediante identificação dos fatores de risco para o desmame precoce na RIL e teve como referencial-base para o desenvolvimento das mensagens educativas os manuais do Ministério da Saúde ${ }^{19-21}$ e as diretrizes da Sociedade Brasileira 
de Pediatria. ${ }^{22,23}$ Após a constituição do corpus do estudo, procedeu-se à análise categorial-temática. ${ }^{24}$

Em seguida, o PMTF foi redigido em linguagem apropriada e as figuras foram confeccionadas por empresa de designer contratada para tal finalidade, sob a supervisão da equipe de pesquisadores. Optou-se pela confecção de figuras que fossem atrativas, de fácil compreensão, condizentes com o contexto sociocultural das nutrizes e que buscassem demonstrar 0 conteúdo das mensagens de texto. ${ }^{25}$ As figuras foram elaboradas no software gráfico Corel Draw X7, de interface Wireframe, com dimensões (1080x1080px - 72dpi) e tamanhos (54pt- 25pt e 17pt) compatíveis com a mídia na qual serão veiculadas.

Quanto à análise de legibilidade, realizou-se a avaliação da adequabilidade da linguagem, por meio do teste de Flesch Kincaid, medida de complexidade do texto associada à facilidade de leitura para diferentes tipos de leitores. O teste foi realizado com a utilização do Revisor Gramatical Automático para o Português ReGra, ferramenta do Microsoft Word, versão 2006, que identifica a estrutura sintática da sentença. $O$ cálculo foi realizado pela seguinte fórmula: IFLC $=206,835-[(1,015 x$ comprimento médio da frase) $+84,6 \times$ (número médio de sílabas)]. Os resultados variam em uma escala de zero a 100 pontos, em que quanto maior a pontuação, mais fácil é o texto. Esse teste foi aplicado, a princípio, em cada mensagem e, subsequentemente, no pacote completo, adotando-se a seguinte referência: índice entre 75100 (textos muito fáceis, adequados para leitores com nível de escolaridade até o quarto ano do ensino fundamental). ${ }^{26}$ Assim, as frases que não atingiram esse índice foram reescritas para facilitar a compreensão do público ao qual se destina (nutrizes alfabetizadas).

Quanto à validação de conteúdo e aparência, esta foi realizada por juízes das áreas de enfermagem e nutrição, com experiência nas temáticas saúde da criança, AM e tecnologias da informação em saúde, os quais decidiram sobre a pertinência das dimensões dos índices de AM. Os juízes necessariamente deveriam ser peritos na área do construto, pois a tarefa consistiu em decidir se os itens estavam se referindo ou não ao traço latente em questão. ${ }^{27}$

Para a definição amostral, realizaram-se consultas em artigos publicados em periódicos de referência nacional e internacional, a fim de localizar os juízes especialistas na temática em tela. Para ser considerado juiz viável, era necessário que atingisse escore mínimo de cinco pontos aos critérios pré-selecionados e adaptados da versão de Fehring. ${ }^{28}$ Em seguida, realizaramse buscas na Plataforma Lattes, do Conselho Nacional de Desenvolvimento Científico e Tecnológico (CNPq) para verificar a adequação dos possíveis participantes aos critérios de inclusão. ${ }^{29} \mathrm{O}$ estabelecimento do tamanho amostral ocorreu por conveniência. Ao todo, selecionaram 48 juízes, dos quais 16 manifestaram interesse em contribuir com o estudo de validação. Após o processo, 11 juízes compuseram a amostra.

Para a coleta de dados, fez-se contato por e-mail e enviouse carta-convite. Após anuência, encaminharam-se, via e-mail, os instrumentos de coleta de dados, as instruções, o Termo de Consentimento Livre e Esclarecido, em duas vias, e uma cópia do PMTF. Empregaram-se três formulários: o primeiro para caracterização dos especialistas, composto de variáveis sociodemográficas e acadêmicas; o segundo, um instrumento para validação de conteúdo, que avalia tecnologias educacionais em seis domínios: objetivos, organização, estilo de escrita, aparência, motivação e adaptação cultural; ${ }^{30}$ e o terceiro, outro formatado por 12 itens, específico para validação de aparência, que avalia materiais educacionais para pessoas com baixo letramento. ${ }^{31}$ Para que os juízes avaliassem a pertinência de cada item, adotou-se escala do tipo Likert, com pontuação de um a cinco, a qual se fundamentou nas respostas dos juízes com relação ao grau de relevância de cada item dos instrumentos.

Após avaliação dos juízes, os dados foram duplamente inseridos em planilha do software Microsoft Excel, versão 2013, depois processados e analisados pelo software Statistical Package for Social Sciences for Windows (SPSS) (2009), versão 18.0. As informações sociodemográficas e acadêmicas foram tabuladas e apresentadas por meio de estatística descritiva, sendo, assim, realizados cálculo de média, frequências relativas e absolutas. Para determinar o nível de concordância entre os juízes quanto ao conteúdo, calculou-se o Índice de Validade de Conteúdo (IVC), verificado a partir do somatório do consentimento dos juízes que marcaram os itens 4 e 5 , divididos pelo número total de respostas. Logo, considerou-se como aceitável índice igual ou superior a $78,0 \%$ (IVC $\geq 0,78$ ), para avaliação individual de cada item e avaliação geral do PMTF. ${ }^{32}$

Para determinar o nível de concordância entre os juízes quanto à aparência, calculou-se o Índice de Validade de Aparência para cada item (IVA-I) computado pelo número de especialistas que responderam 4 ou 5 dividido pelo total de especialistas que avaliaram o item. Para o IVA total (IVA-T), realizou-se a soma dos IVA-I e dividido pelo total de itens. ${ }^{31}$ Considerou-se com IVA $>0,78$ excelente, àqueles com IVA $<0,78$ e necessidades de adequação na aparência da tecnologia educacional em saúde. ${ }^{31}$

Para mostrar a validade convergente do PMTF, os resultados do instrumento de avaliação de conteúdo e do instrumento de avaliação de aparência foram sumarizados por escore, mediante somatório das respostas das escalas. A técnica de convergência utilizada foi conduzida com base na correlação entre os escores de duas escalas, cujos conteúdos estão direcionados para o mesmo sentido teórico. ${ }^{33}$ Procedeu-se ao cálculo de correlação de Pearson, dos escores total e dos domínios do instrumento de validação de conteúdo, bem como do escore do instrumento de validação de aparência. Consideraram-se os resultados de correlações acima de $r>0,3$ e $p<0,05$ como sendo validação de convergência plausível. O nível de significância adotado foi de $p \leq 0,05$, com Intervalo de Confiança (IC) de $95,0 \%$ para os testes estatísticos realizados.

Estudo aprovado pelo Comitê de Ética em Pesquisa do Campus Amílcar Ferreira Sobral, da Universidade Federal do Piauí, conforme Certificado de Apresentação para Apreciação Ética noㅜ 03637218.7.0000.5660 e parecer $\mathrm{n}$ ㅇ 3.055.341. 


\section{RESULTADOS}

A versão final da tecnologia educativa desenvolvida possuiu quantitativo de 28 mensagens de texto e respectivas figuras, organizadas em blocos, seguindo sequência lógica de agrupamento por temas comuns: Bloco 1 - Apresentação e finalização do acompanhamento; Bloco 2 - Introdução sobre o AM; Bloco 3 - Vantagens do AM; Bloco 4 - Posição e pega; Bloco 5 - Evolução e produção do leite materno; Bloco 6 - Cuidados com as mamas; Bloco 7 - Ordenha do leite; Bloco 8 - Possíveis complicações na mama; Bloco 9 - Apoio profissional; Bloco 10 - Instrumentos legais de proteção ao AM; e Bloco 11 - Oferta de líquidos.

Uma vez construído o PMTF, realizou-se o teste de legibilidade de Flesch Kincaid em cada uma das 28 mensagens de texto, obtendo-se variação de índice entre 75 e 99 e no pacote completo com média de 87 pontos. Deste modo, verificou-se que a linguagem empregada na tecnologia educacional era adequada para indivíduos com baixo nível de escolaridade e/ ou que não tinham o hábito de ler. Exemplo das mensagens desenvolvidas e respectiva imagem é apresentado na Figura 1.

A amostra foi composta de 11 juízes, dez enfermeiras e um nutricionista. As idades dos juízes variaram entre 30 e 50 anos. Dos 11 juízes selecionados, dois $(18,2 \%)$ concluíram pós-doutorado na área de Enfermagem Obstétrica, oito (72,7\%) apresentavam doutorado em Enfermagem, Enfermagem em Saúde da Criança, do Adolescente e em Pediatria. Os juízes eram docentes e publicaram trabalhos na área do estudo em tela (100,0\%). O tempo médio de formação foi 33,36 anos, sendo a maioria (oito - 72,7\%) entre um a 20 anos. Sobre as regiões do Brasil nas quais os juízes trabalhavam, verificou-se que participaram da pesquisa juízes de quatro regiões brasileiras (Norte, Nordeste, Sul e Sudeste) e de seis estados (Piauí, Ceará, São Paulo, Pará, Paraná, Rio Grande do Sul).
Com relação à validação do PMTF, observou-se média do IVC superior a 0,86 nas cinco dimensões do instrumento avaliadas. O IVC global da tecnologia desenvolvida foi de 0,86, sendo, portanto, satisfatório e permitindo considerar o PMTF validado quanto ao conteúdo (Tabela 1). Da mesma forma, o IVA de 0,85 admite considerar o pacote válido quanto à aparência.

Inicialmente, os juízes avaliaram o PMTF quanto aos objetivos a serem atingidos com a utilização. Nesse domínio, os itens foram considerados válidos, visto que houve concordância mínima de 82,0\% (IVC 0,82). Dentre os itens alusivos à dimensão organização, a menor concordância foi de 73,0\% (IVC 0,73) naquele relacionado à ordenação das mensagens. Dois juízes discordaram desse item e um não concordou e nem discordou. Os juízes discordantes sugeriram mudanças na sequência de apresentação das mensagens, de modo que aspectos relacionados à descida do leite e às fissuras mamilares fossem orientados nas primeiras mensagens. Optou-se por acatar as sugestões dos especialistas e, deste modo, a sequência das mensagens/ figuras foi modificada.

Ainda, relativo à dimensão organização, os itens "o material via plataforma virtual está adequado" e "o número de mensagens está adequado" apresentaram concordância entre os juízes de $91,0 \%$, indicando que os especialistas concordaram que o PMTF é válido como tecnologia educativa para ser apresentada via mídia social (Whatzapp $®$ Messenger) e que a opção pelo envio de mensagens diariamente, durante o período neonatal (28 dias), é suficiente para atender à proposta de colaborar na implementação e manutenção do AM nessa fase.

Quanto à dimensão estilo de escrita, houve 91,0\% de concordância dos juízes nos itens "o texto é vívido e interessante/o tom é amigável" e "o vocabulário é acessível". Um juiz discordou e outro não concordou e nem discordou. Aquele que discordou apresentou como justificativa a necessidade de mudança de alguns

\begin{tabular}{|l|l|l|}
\hline Imagen & \multicolumn{1}{|c|}{ Mensagem } & \multicolumn{1}{|c|}{$\begin{array}{c}\text { Teste de } \\
\text { legibilidade de } \\
\text { Flesch Kincaid }\end{array}$} \\
\hline & $\begin{array}{l}\text { Logo que o bebê nasce, o leite } \\
\text { materno costuma ser pouco. Esse } \\
\text { leite é chamado colostro, tem cor } \\
\text { amarela e funciona como a primeira } \\
\text { vacina do bebê, pois o protege } \\
\text { contra várias doenças. Este é o leite } \\
\text { ideal e naito medida certa para } \\
\text { alimentar o bebê nos primeiros dias } \\
\text { de vida. Após cerca de 3 a 5 dias, a } \\
\text { produção de leite irá aumentar. }\end{array}$ \\
\hline
\end{tabular}

Figura 1. Exemplo de mensagem e respectiva figura a ser enviada às nutrizes para promoção do aleitamento materno, no período neonatal. Floriano, PI, Brasil, 2020 
termos, como substituir a expressão fissuras nos mamilos por frase mais popular: feridas nos mamilos. Essa e outras sugestões que seguiram a mesma pauta foram acatadas. No entanto, de acordo com as possibilidades e a fim de alcançar os maiores índices relativos ao teste de legibilidade, buscou-se manter as duas expressões, popular e técnica, explicando-se mutuamente, com o intuito de atingir o objetivo completo da tecnologia educativa, que também inclui elevar o letramento das nutrizes.

Tabela 1. Índice de validade de conteúdo e aparência do pacote de mensagens de texto e figuras, segundo a análise dos juízes. Floriano, PI, Brasil, 2020

\begin{tabular}{|c|c|c|}
\hline \multirow{2}{*}{ Dimensões do instrumento de validação de conteúdo } & \multicolumn{2}{|c|}{ Concordância dos juízes } \\
\hline & n (\%) & IVC \\
\hline 1. Dimensão objetivo & $29(88)$ & 0,88 \\
\hline 1.1Atende ao objetivo geral da pesquisa. & $9(82)$ & 0,82 \\
\hline $\begin{array}{l}\text { 1.2 Ajuda na aquisição e na troca de conhecimentos entre o profissional de saúde e de } \\
\text { nutrizes em aleitamento materno. }\end{array}$ & $10(91)$ & 0,91 \\
\hline $\begin{array}{l}\text { 1.3 Está adequado para ser usado por qualquer profissional que trabalhe na Estratégia Saúde } \\
\text { da Família. }\end{array}$ & $10(91)$ & 0,91 \\
\hline 2. Dimensão organização & $57(86)$ & 0,86 \\
\hline 2.1 A mensagem inicial está atraente, indica a finalidade do contato. & $9(82)$ & 0,82 \\
\hline 2.2 O tamanho do título da mensagem dentro do material está adequado. & $9(82)$ & 0,82 \\
\hline 2.3 As mensagens seguem uma ordem. & $8(73)$ & 0,73 \\
\hline 2.4 O material via plataforma virtual está adequado. & $10(91)$ & 0,91 \\
\hline 2.50 número de mensagens está adequado. & $10(91)$ & 0,91 \\
\hline 2.6 Os temas retratam aspectos-chave importantes. & $11(100)$ & 1,00 \\
\hline 3. Dimensão estilo de escrita & $57(86)$ & 0,86 \\
\hline 3.1 A escrita está em um estilo adequado à nutriz em aleitamento materno. & $9(82)$ & 0,82 \\
\hline 3.20 texto é vívido e interessante. O tom é amigável. & $10(91)$ & 0,91 \\
\hline 3.3 O vocabulário é acessível. & $10(91)$ & 0,91 \\
\hline 3.4 Há associação do tema de cada sessão com o texto correspondente. & $11(100)$ & 1,00 \\
\hline 3.5 O texto está claro. & $9(82)$ & 0,82 \\
\hline 3.6 O estilo da redação correspondente ao nível de conhecimento do público-alvo. & $8(73)$ & 0,73 \\
\hline 4. Dimensão aparência & $38(86)$ & 0,86 \\
\hline 4.1 As mensagens parecem organizadas. & $9(82)$ & 0,82 \\
\hline 4.2 As figuras são simples. & $11(100)$ & 1,00 \\
\hline 4.3 As figuras servem para complementar os textos. & $9(82)$ & 0,82 \\
\hline 4.4 As figuras são expressivas e suficientes. & $9(82)$ & 0,82 \\
\hline 5. Dimensão motivação & $57(86)$ & 0,86 \\
\hline 5.10 material está apropriado para idade, gênero e cultura. & $10(91)$ & 0,91 \\
\hline 5.20 material apresenta lógica. & $9(82)$ & 0,82 \\
\hline 5.3 A interação é convidada pelo texto e sugere ações. & $8(73)$ & 0,73 \\
\hline 5.4 Aborda assuntos necessários à nutriz em aleitamento materno. & $11(100)$ & 1,00 \\
\hline 5.5 Promove mudança de comportamento e atitude. & $8(73)$ & 0,73 \\
\hline 5.60 material propõe à nutriz em aleitamento materno a realização do autocuidado. & $11(100)$ & 1,00 \\
\hline Índice de Validade de Conteúdo & & 0,86 \\
\hline Índice de Validade de Aparência total* & & 0,85 \\
\hline
\end{tabular}

*Os IVA-I foram suprimidos. 
Ainda nessa dimensão, o item "o estilo da redação correspondente ao nível de conhecimento do público-alvo" atingiu concordância inferior a $80,0 \%$. Porém, enfatiza-se que um juiz discordou parcialmente dessa afirmativa (dois outros participantes não concordaram nem discordaram) e que, anteriormente ao encaminhamento do PMTF aos juízes, o material foi submetido ao teste de legibilidade de Flesch Kincaid, atingindo índices superiores a 75 , que classificam as mensagens produzidas como "Muito Fácil". No entanto, como referido, algumas sugestões foram acatadas para facilitar a compreensão do conteúdo das mensagens pelo público-alvo.

Sobre a dimensão aparência, dos 11 juízes, nove (82,0\%) concordaram que as mensagens pareciam organizadas, que as figuras serviam para complementar os textos e que estas eram expressivas e suficientes. Deste modo, alguns juízes sugeriram reorganização da escrita de trechos avaliados como pouco informativos. Logo, modificou-se a frase "Então, converse com seus familiares" para "Então, converse com sua família, amigos e pessoas próximas, caso se sinta sozinha". No que concerne à dimensão motivação, houve concordância mínima de 73,0\% acerca da interação convidada pelo texto, sugestão de ações e promoção de mudança de comportamento e atitude. Para $91,0 \%$ e $82,0 \%$ dos juízes, o PMTF, respectivamente, estava apropriado para idade, gênero e cultura, bem como apresentava lógica.

Ademais, houve unanimidade de concordância dos juízes nos itens do PMTF, referentes à apresentação de temas que retratam aspectos-chave importantes (dimensão organização); associação do tema de cada sessão com o texto correspondente (dimensão estilo de escrita); exposição de figuras simples (dimensão aparência); abordagem de assuntos necessários à nutriz em $\mathrm{AM}$; proposta à nutriz em $\mathrm{AM}$ para realização do autocuidado, ambos da dimensão motivação.

Portanto, ao considerar os valores do IVC das respostas dos itens do questionário de validação de conteúdo dos juízes participantes, percebeu-se que dos 25 itens avaliados, 24 foram considerados válidos. Contudo, o item 5.5 (Promove mudança de comportamento e atitude) não obteve IVC satisfatório. Acerca das figuras, algumas modificações também foram solicitadas e acatadas pela equipe de pesquisadores, como alteração da coloração da letra utilizada, remoção de tópicos presentes no corpo da figura para diminuição do excesso de informação nas imagens, entre outras.

Na Tabela 1, observa-se o IVA do PMTF, classificado como excelente $(0,85)$, evidenciando que o material produzido contempla ilustrações e textos dispostos visualmente em layout que favorece a comunicação e a educação para saúde. Os juízes apresentaram comentários sobre a tecnologia produzida, a proposta de o PMTF auxiliar mulheres e famílias a ter sucesso no processo de AM.

Com a finalidade de assegurar outros elementos de validade para o PMTF, a Tabela 2 expõe os resultados da validade convergente.

A Tabela 2 mostra que o PMTF apresentou convergência entre os escores total e os domínios do instrumento de
Tabela 2. Validade convergente do pacote de mensagens de texto e figuras para promoção do aleitamento materno no período neonatal. Floriano, $\mathrm{PI}$, Brasil, 2020

\begin{tabular}{lcc}
\hline $\begin{array}{c}\text { Domínios } \\
\text { Instrumento de } \\
\text { Validação de } \\
\text { Conteúdo }\end{array}$ & $\begin{array}{c}\text { Instrumento de Validação } \\
\text { de Aparência de Tecnologia } \\
\text { Educacional }\end{array}$ & p-valor* \\
\hline Objetivos & $0,281^{*}$ & 0,402 \\
\hline Organização & $0,713^{*}$ & 0,014 \\
\hline Estilo de Escrita & $0,697^{*}$ & 0,017 \\
\hline Aparência & $0,392^{*}$ & 0,233 \\
\hline Motivação & $0,588^{*}$ & 0,057 \\
\hline Total SAM & $0,735^{*}$ & 0,010 \\
\hline
\end{tabular}

*Coeficiente de Correlação de Pearson

validade de conteúdo com os escores de aparência, revelando a validade convergente do material. Os índices de correlação do instrumento de validade de aparência foram fortes com os domínios organização, estilo de escrita e motivação, correlação moderada com a aparência e fraca com o domínio objetivos. No entanto, este último não atingiu o critério adotado de correlação acima de 0,3 para a validade convergente. Esses resultados mostram que o conteúdo e a aparência do PMTF estiveram na mesma direção de qualidade e foram condizentes com a literatura sobre $o$ assunto.

\section{DISCUSSÃO}

O conteúdo que embasou a construção do PMTF para a promoção do aleitamento materno no período neonatal contemplou aspectos relacionados com: descida do leite materno; tipos de mamilo; produção do leite materno; técnica de amamentação (posição e pega); definição, prevenção e manejo de fissuras mamilares; definição, prevenção e manejo do ingurgitamento mamário e da mastite; percepções acerca de leite fraco/pouco leite; dor ao amamentar; conhecimento profissional e influências do contexto sociofamiliar na manutenção do AME; instrumentos legais de proteção ao AM no Brasil; oferta de fórmulas infantis/ leites artificiais e influência na produção do leite materno; oferta de outros líquidos e influência na produção do leite materno; uso de mamadeira e chupeta, entre outros.

Esses conteúdos foram relevantes ao material educativo, pois, como identificado na revisão integrativa realizada, podem constituir fatores de proteção ao AM ou de risco para o desmame precoce. Assim, evidenciou-se que a percepção das nutrizes, muitas vezes equivocada, de demora para a descida do leite, ${ }^{34}$ além da presença de mamilos planos ou invertidos, ${ }^{35}$ bem como a presença de lesões mamilares ${ }^{36} e$ de dor associada ao ato de amamentar ${ }^{37}$ podem desencadear o desmame precoce. Ademais, a técnica da amamentação ineficaz, que dificulta a sucção e o esvaziamento da mama, podendo gerar prejuízos na dinâmica 
da produção de leite e problemas da mama, como fissuras, ingurgitamento mamário e mastite (mais comuns no período neonatal), também pode ser associada à interrupção do AME. ${ }^{38}$

O conteúdo utilizado na construção do material educativo contemplou, também, o uso de bicos artificiais (mamadeira e chupeta), considerados intervenientes que podem incrementar o risco de desmame precoce ${ }^{39}$ além de fórmula infantil e leite artificial, ambos considerados preditores de tempos mais curtos de AM. ${ }^{40}$ Em contrapartida, o apoio e a promoção familiar e dos profissionais de saúde se configuram como fatores de proteção ao $\mathrm{AM}$, mostrando que a prática do $\mathrm{AM}$ é influenciada pelo meio em que a nutriz se encontra e pelas atitudes dos significantes, como maridos/companheiros e avós da criança, que se somam às da nutriz, diante da disponibilidade para a lactação. ${ }^{41}$

Mesmo com construção baseada em evidências científicas, foi importante a validação de conteúdo e aparência do PMTF para ancoragem científica e credibilidade da tecnologia educativa. ${ }^{42}$ Os juízes que validaram o conteúdo eram, na maioria, enfermeiros, cujos dados corroboram outros estudos de validação de materiais educativos que envolvem promoção do AM, justificado pelo fato de que, em geral, o enfermeiro é o profissional com papel relevante na aplicação de tecnologias e intervenções educativas para o alcance de melhores taxas de $\mathrm{AM},{ }^{18,43}$ além de ser profissional apto a identificar e diagnosticar situações desfavoráveis ao AM. ${ }^{18}$

Outro dado significativo sobre o perfil dos juízes participantes diz respeito à procedência. Esse aspecto demonstrou que o PMTF foi validado por profissionais de diferentes regiões e estados brasileiros. Tal característica representa aspecto favorável, visto que foi possível reunir diversos saberes especializados na temática abordada pelo material, em especial, pelo contexto brasileiro que apresenta dimensões continentais e, consequentemente, diferentes experiências, práticas e perspectivas acerca do AM. ${ }^{44}$

Conforme as avaliações realizadas pelos juízes, o PMTF foi validado em 24 dos 25 itens do questionário. Assim, algumas modificações foram solicitadas, com o intuito de incluir assuntos relevantes que, na perspectiva dos juízes, poderiam contribuir para a implementação e manutenção do AM no período neonatal, em especial, no que diz respeito ao enfrentamento dos principais fatores que contribuem para o desmame precoce nessa fase. Enfatiza-se que não foi sugerido pelos juízes a retirada de mensagens ou figuras apresentadas no pacote. ${ }^{45}$

Com a validação do material, os juízes evidenciaram que o conteúdo das mensagens apresenta clareza para o alcance do objetivo proposto que envolve melhorar o desempenho de nutrizes no processo de AM. Para os juízes, a tecnologia produzida colabora na aquisição e troca de conhecimentos entre profissional de saúde e nutrizes em aleitamento. Sobre isso, pesquisa de revisão sistemática, com vistas a fornecer percepção abrangente das intervenções digitais existentes que apoiam o AM, evidenciou que a eficácia dos sistemas de informação e comunicação para combater barreiras ao AM foi demonstrada predominantemente em termos de conhecimento aprimorado sobre a temática. Elucidou-se, ainda, que o nível de conhecimentos das nutrizes pode ajudá-las em diversos aspectos como evitar desconforto físico e perceber o suprimento de leite. ${ }^{17}$

Os juízes também concordaram com a questão que as mensagens estavam bem organizadas e as figuras completavam o sentido dos textos, além de serem simples e expressivas. Buscou-se emprego adequado de ilustrações, a fim de permitir a legibilidade e compreensão das mensagens apresentadas, as quais foram propostas como forma de atrair as leitoras, despertar e manter o interesse pela leitura, complementar e reforçar a informação fornecida pelo profissional de saúde em relação ao processo de AM. Salienta-se, assim, que as figuras devem fazer parte do material educativo para facilitar o entendimento do leitor e, para isso, precisam contemplar personagens, cenários e vivências mais próximas do público-alvo, possibilitando a oportunidade de construir novos significados e permitindo a compreensão do cotidiano. ${ }^{46} \mathrm{O}$ emprego de figuras associadas a texto faz com que o material educativo seja de adequada compreensão durante a leitura. ${ }^{47}$

Quanto à dimensão motivação, um dos itens não atingiu a validação, concernente à possibilidade de a tecnologia produzida promover mudança de comportamento e atitude entre as nutrizes durante o processo de amamentação. De fato, essa limitação havia sido apontada por alguns juízes participantes e, também, encontra respaldo em outros estudos de validação de materiais educacionais, os quais, por sua vez, encontraram resultados semelhantes. ${ }^{31,48,49}$ Isto sugere que essa dimensão do instrumento precisa ser reavaliada quanto à pertinência para validação de conteúdo.

É preciso refletir e reconhecer que, quanto aos aspectos relacionados à saúde e aos hábitos de vida, a mudança não ocorre repentinamente, mas carece que o sujeito esteja disposto a mudar. ${ }^{49}$ De modo similar, os juízes refletiram sobre a complexidade envolvendo mudanças comportamentais e as possíveis limitações do PMTF que, isoladamente, não teria capacidade de promover mudanças sem o devido acompanhamento individual. ${ }^{48}$ Contudo, destaca-se que está prevista no protocolo desta pesquisa a realização de estudo quase-experimental que terá como público-alvo nutrizes em processo de AM no período neonatal, correspondendo à segunda etapa deste trabalho, em que se investigará a efetividade da tecnologia educacional avaliada neste estudo.

Concorda-se que a informação fornecida por meio da ferramenta Whatzapp $®$ Messenger, pode não ser suficiente para modificar os comportamentos das nutrizes. No entanto, contribui para a construção de conhecimento, fator necessário para a adesão ao AM ou a qualquer outro hábito de saúde. ${ }^{50}$ Destacase, ainda, que a avaliação dos juízes ocorreu anteriormente ao período da pandemia do novo coronavírus, de modo que se pode considerar a importância do PMTF como ferramenta de trabalho que, além de possibilitar educação em saúde, mantém medidas preventivas de distanciamento social.

Isso posto, e a partir da compreensão de que itens como: "ajuda na aquisição e na troca de conhecimentos entre o profissional de saúde e de nutrizes em aleitamento materno" 
(IVC 0,91) e "o material propõe à nutriz em aleitamento materno a realização do autocuidado" (IVC 1,00) atingiram índices de validade de conteúdo excelentes, pode-se inferir que o PMTF poderá impactar de modo positivo a promoção do AM.

Ademais, é importante considerar que a proposta de aplicação da intervenção educativa é precoce, iniciando, ainda, no Alojamento Conjunto da maternidade. À vista disto, metanálise realizada recentemente, com o objetivo de descrever as evidências publicadas de intervenções, visando promover o AM exclusivo entre as mulheres no pós-parto, em termos de suas características, indicou que intervenções com a finalidade de promover o AM, sob a perspectiva de mudanças comportamentais, devem ser iniciadas durante a primeira semana pós-parto, ou antes. O que tende a ser um período de adaptação e momento em que a maioria das mulheres consegue se concentrar no AM. Apontase, ainda, que ao iniciar a intervenção, deve-se considerar o fato de que as mulheres podem ser menos propensas a amamentar se perderem a oportunidade logo após o nascimento do bebê. ${ }^{51}$

Em relação à validade convergente, os resultados encontrados foram promissores e mostram pacote de mensagens de texto e figuras com coerência teórico-conceitual, dispostas em estrutura de layout favorável às atividades as quais se destinam. Os escores do instrumento de validação de aparência mostraram índice de correlação total moderado com o instrumento de validação de conteúdo, demarcando a validade convergente do PMTF. Apesar de ser um desafio para os estudos de validação em virtude da dificuldade na escolha dos instrumentos para comparação, a fim de demonstrar coerência e convergência dentro do material que está sendo avaliado, os resultados encontrados sinalizaram diferencial nas evidências de validade do material desenvolvido neste estudo.

A escolha pelos escores de validação de conteúdo e aparência para realizar o estudo de validação de convergência é um dos pontos fortes do estudo, uma vez que não foram encontradas pesquisas de construção e validação interna de materiais educacionais que trouxessem tais análises. Esta escolha ocorreu pela ligação entre os conceitos de conteúdo, aparência e a representação de ambos na elaboração de tecnologias educacionais demonstrada em outros estudos..$^{31,44}$

\section{CONCLUSÕES E IMPLICAÇÕES PARA A PRÁTICA}

O Pacote de Mensagens de Texto e Figuras foi construído com consistente embasamento científico, desde a escolha do momento de aplicação (período neonatal) até os aspectos abordados nas mensagens. $O$ teste de legibilidade se mostrou satisfatório para impedir barreiras de aprendizado em decorrência da baixa escolaridade. A tecnologia educativa apresentou indícios de validade de conteúdo, aparência e convergência, mostrando qualidade e potencial para ser efetivo na promoção do aleitamento materno no período neonatal.

Trata-se de tecnologia educativa de baixo custo e que poderá ser usada por meio do Whatzapp $\AA$ Messenger para se aproximar das nutrizes, apoiando-as para reagir frente aos problemas comuns nesse período, e que, estatisticamente, estão associados aos índices de desmame precoce. Conjectura-se que o seu uso poderá ser realizado no âmbito da Estratégia Saúde da Família pelos enfermeiros e agente comunitário de saúde que mantém em suas áreas adscritas o uso de aplicativos de mensagens para aproximar a comunidade ao serviço de saúde.

Como limitações, elencam-se o reduzido número de juízes e a não realização de piloto para validação semântica. Porém, quanto à restrição relativa aos juízes, enfatiza-se que a constituição de um corpo de juízes de alto nível e proveniente de diversas regiões do país conseguiu equalizar o quantitativo restrito e, deste modo, elevar a qualidade das análises do Pacote de Mensagens de Texto e Figuras.

\section{FINANCIAMENTO}

Apoio financeiro do Conselho Nacional de Desenvolvimento Científico e Tecnológico (CNPq), Brasil, processo no 420768/20181, concedido a Maria Augusta Rocha Bezerra.

\section{CONTRIBUIÇÕES DOS AUTORES}

Desenho do estudo. Mariana Mesquita Silva. Maria Augusta Rocha Bezerra

Coleta ou produção dos dados. Mariana Mesquita Silva Análise de dados. Mariana Mesquita Silva. José Wicto Pereira Borges. Jardeliny Correa da Penha. Maria Augusta Rocha Bezerra Interpretação dos resultados. Mariana Mesquita Silva. José Wicto Pereira Borges. Cristianne Teixeira Carneiro. Izabel Cristina Falcão Juvenal Barbosa. Maria Augusta Rocha Bezerra

Redação e revisão crítica do manuscrito. Mariana Mesquita Silva. Cristianne Teixeira Carneiro. Jardeliny Correa da Penha. Izabel Cristina Falcão Juvenal Barbosa. José Wicto Pereira Borges. Maria Augusta Rocha Bezerra

Aprovação da versão final do artigo. Mariana Mesquita Silva. Cristianne Teixeira Carneiro. Jardeliny Correa da Penha. Izabel Cristina Falcão Juvenal Barbosa. José Wicto Pereira Borges. Maria Augusta Rocha Bezerra.

Responsabilidade por todos os aspectos do conteúdo e a integridade do artigo publicado. Mariana Mesquita Silva. Cristianne Teixeira Carneiro. Jardeliny Correa da Penha. Izabel Cristina Falcão Juvenal Barbosa. José Wicto Pereira Borges. Maria Augusta Rocha Bezerra.

\section{EDITOR ASSOCIADO}

Eliane Tatsch Neves

\section{REFERÊNCIAS}

1. Cherrez-Ojeda I, Vanegas E, Felix M, Mata VL, Jiménez FM, Sanchez $M$ et al. Frequency of use, perceptions and barriers of information and communication technologies among Latin American physicians: an ecuadorian cross-sectional study. J Multidiscip Healthc. 2020 mar; 13:25969. http://dx.doi.org/10.2147/JMDH.S246253. PMid:32214821. 
2. Huo J, Desai R, Hong YR, Turner K, Mainous 3rd AG, Bian J. Use of social media in health communication: findings from the Health Information National Trends Survey 2013, 2014, and 2017. Cancer Contr. 2019 jan-dez;26(1):1073274819841442. http://dx.doi. org/10.1177/1073274819841442. PMid:30995864.

3. Formagini TDB, Ervilha RR, Machado NM, Andrade BABB, Gomide HP, Ronzani TM. Revisão dos aplicativos de smartphones para cessação do tabagismo disponíveis em língua portuguesa. Cad Saude Publica. 2017 mar;33(2):e00178215. http://dx.doi.org/10.1590/0102-311x00178215. PMid:28300972.

4. Alianmoghaddam N, Phibbs S, Benn C. "I did a lot of Googling": a qualitative study of exclusive breastfeeding support through social media. Women Birth. 2019 abr;32(2):147-56. http://dx.doi.org/10.1016/j. wombi.2018.05.008. PMid:29921552.

5. Lima ICV, Galvão MTG, Pedrosa SC, Cunha GH, Costa AKB. Use of the WhatsApp application in health follow-up of people with HIV: a thematic analysis. Esc Anna Nery. 2018 abr;22(3):e20170429. http:/l dx.doi.org/10.1590/2177-9465-ean-2017-0429.

6. Chari A, Gane SBC. Aplicativos de mensagens instantâneas na área da saúde: estamos aproveitando seu potencial? BMJ Innov. 2018;4(1):5-8. http://dx.doi.org/10.1136/bmjinnov-2017-000197.

7. Caetano R, Silva AB, Guedes ACCM, Paiva CCN, Ribeiro GR, Santos DL et al. Challenges and opportunities for telehealth during the COVID-19 pandemic: ideas on spaces and initiatives in the Brazilian context. Cad Saude Publica. 2020;36(5):e00088920. http://dx.doi.org/10.1590/0102311x00088920. PMid:32490913.

8. Nascimento MHM, Teixeira E. Tecnologia educacional para mediar o acolhimento de "familiares cangurus" em unidade neonatal. Rev Bras Enferm. 2018;71(Supl.3):1290-7. http://dx.doi.org/10.1590/0034-71672017-0156. PMid:29972526.

9. Dewanti LP, Februhartanty J, Roshita A. The new way of peer support for improving breastfeeding performance. J Health Educ. 2019;4(1):23-8. http://dx.doi.org/10.15294/jhe.v4i1.29407.

10. Marcon AR, Bieber M, Azad MB. Protecting, promoting, and supporting breastfeeding on Instagram. Matern Child Nutr. 2019;15(1):e12658. http://dx.doi.org/10.1111/mcn.12658. PMid:30079555.

11. Farr R, Rahman F, O'Riordan MA, Furman L. Assessing the feasibility and effectiveness of two prenatal breastfeeding. Breastfeed Med. 2019 dez;14(10):724-30. http://dx.doi.org/10.1089/bfm.2019.0053. PMid:31657635.

12. Santos AF, Fonseca SD, Araujo LL, Procópio CSD, Lopes EAS, Lima AMLD et al. Incorporação de Tecnologias de Informação e Comunicação e qualidade na atenção básica em saúde no Brasil. Cad Saude Publica. 2017 jun;33(5):1-14. http://dx.doi.org/10.1590/0102-311x00172815. PMid:28640330.

13. Victora CG, Bahl R, Barros AJD, França GVA, Horton S, Krasevec $\mathrm{J}$ et al. Breastfeeding in the 21st century: epidemiology, mechanisms, and lifelong effect. Lancet. 2016 jan;387(10017):475-90. http://dx.doi. org/10.1016/S0140-6736(15)01024-7. PMid:26869575.

14. Covid-19: OMS cria canal com mensagens informativas pelo WhatsApp Agência Brasil [Internet]. 2020 abr 15 [citado 20 maio 2020]. Disponível em: https://agenciabrasil.ebc.com.br/saude/noticia/2020-04/covid-19oms-cria-canal-com-mensagens-informativas-pelo-whatsapp

15. Machado RCG, Turrini RNT, Sousa CS. Mobile applications in surgical patient health education: an integrative review. Rev Esc Enferm USP. 2020 mar;54:e03555. http://dx.doi.org/10.1590/s1980-220x2018032803555 PMid:32236350.

16. Woods J, Moorhouse M, Knight L. A descriptive analysis of the role of a WhatsApp clinical discussion group as a forum for continuing medical education in the management of complicated HIV and TB clinical cases in a group of doctors in the Eastern Cape, South Africa. South. South Afr J HIV Med. 2019 ago;20(1):982. http://dx.doi.org/10.4102/sajhivmed. v20i1.982. PMid:31534790.

17. Tang K, Gerling K, Chen W, Geurts L. Information and communication systems to tackle barriers to breastfeeding: systematic search and review. J Med Internet Res. 2019 set;21(9):e13947. http://dx.doi. org/10.2196/13947. PMid:31573903.

18. Silva NVN, Pontes CM, Sousa NFC, Vasconcelos MGL. Health technologies and their contributions to the promotion of breastfeeding: an integrative review of the literature. Cien Saude Colet. 2019 fev;24(2):589-602. http:// dx.doi.org/10.1590/1413-81232018242.03022017. PMid:30726390.

19. Ministério da Saúde (BR), Fundo das Nações Unidas para a Infância, Organização Mundial da Saúde. Iniciativa Hospital Amigo da Criança: revista, atualizada e ampliada para o cuidado integrado: módulo 2 : fortalecendo e sustentando a iniciativa hospital amigo da criança: um curso para gestores. Brasília: Ministério da Saúde; 2009.

20. Ministério da Saúde (BR), Secretaria de Atenção à Saúde. Estratégia Nacional para Promoção do Aleitamento Materno e Alimentação Complementar Saudável no Sistema Único de Saúde: manual de implementação. Brasília: Ministério da Saúde; 2015.

21. Ministério da Saúde (BR), Secretaria de Atenção à Saúde, Departamento de Atenção Básica. Saúde da criança: aleitamento materno e alimentação complementar. Brasília: Ministério da Saúde; 2015.

22. Sociedade Brasileira de Pediatria, Departamento Científico de Aleitamento Materno. Amamentação: a base da vida. São Paulo: SBP; 2018.

23. Sociedade Brasileira de Pediatria, Departamento Científico de Aleitamento Materno. Uso de chupeta em crianças amamentadas: prós e contras. São Paulo: SBP; 2017.

24. Bardin L. Análise de conteúdo. 4. ed. Lisboa: Edições 70; 2011.

25. Doak CC, Doak LG, Root J. Teaching patients with low literacy skills. Philadelphia: J.B. Lippincott; 1996.

26. Martin TBF, Ghiraldelo CM, Nunes MGV, Oliveira Jr ON. Readability formulas applied to textbooks in brazilian portuguese. São Carlos: Instituto ed; 1996.

27. Borges JWP, Moreira TMM, Rodrigues MTP, Souza ACC, Silva DB. Content validation of the dimensions constituting non-adherence to treatment of arterial hypertension. Rev Esc Enferm USP. 2013 out;47(5):1076-2. http://dx.doi.org/10.1590/S0080-623420130000500010. PMid:24346446.

28. Lira ALBC, Lopes MVO. Nursing diagnosis: educational strategy based on problem-based learning. Rev Lat Am Enfermagem. 2011 ago;19(4):936-43. http://dx.doi.org/10.1590/S0104-11692011000400012. PMid:21876946.

29. Wild CF, Nietsche EA, Salbego C, Teixeira E, Favero NB. Validação de cartilha educativa: uma tecnologia educacional na prevenção da dengue. Rev Bras Enferm. 2019 out;72(5):1318-25. http://dx.doi. org/10.1590/0034-7167-2018-0771. PMid:31531657.

30. Sousa CS, Turrini RNT. Creating and validating educational material for patients undergoing orthognathic surgery. Asian Nurs Res. 2012 dez;6(4):166-72. http://dx.doi.org/10.1016/j.anr.2012.10.006. PMid:25031119.

31. Souza AC, Borges JWP, Moreira TMM. Development of tool to validate the appearance of educational technology in health. Rev Bras Enferm 2020;73(Supl 6).

32. Polit DF, Beck CT, Owen SV. Is the CVI an acceptable indicator of content validity appraisal and recommendations. Res Nurs Health. 2007 ago;30(4):459-67. http://dx.doi.org/10.1002/nur.20199. PMid:17654487.

33. Boateng GO, Neilands TB, Frongillo EA, Melgar-Quiñonez HR, Young SL. Best practices for developing and validating scales for health, social, and behavioral research: a primer. Front Public Health. 2018 jun;6:149. http://dx.doi.org/10.3389/fpubh.2018.00149. PMid:29942800.

34. Medeiros TML, Marcelino JFQ. Percepção de puérperas sobre o seu desempenho ocupacional no pós-operatório da cesáriana. Cad Brase Terap Ocupacional. 2018;26(1):97-109. http://dx.doi.org/10.4322/25268910.ctoAO0960.

35. Urbanetto PDG, Costa AR, Gomes GC, Nobre CMG, Xavier DM, Jung $B C$. Facilities and difficulties found by mothers to breastfeed. Rev Pesqui Cuid Fundam. 2018 jun;10(2):399-405. http://dx.doi.org/10.9789/21755361.2018.v10i2.399-405.

36. Barbosa GEF, Silva VB, Pereira JM, Soares MS, Medeiros Fo RA, Pereira LB et al. Dificuldades iniciais com a técnica da amamentação e fatores associados a problemas com a mama em puérperas. Rev Paul Pediatr. 2017 set;35(3):265-72. http://dx.doi.org/10.1590/19840462/;2017;35;3;00004. PMid:28977289.

37. Costa AMPC, Cavalcante LMS, Araujo DN, Santos ACS. Breastfeeding and intercurrences that contribute to early weaning: an integrative review. Int J Dev Res [Internet]. 2019; [citado 2020 jun 26];9(6):28048- 
52. Disponível em: https://www.journalijdr.com/breastfeeding-andintercurrences-contribute-early-weaning-integrative-review

38. Almeida JM, Martins ACV, Amaral DM, Batista HP, Almeida LCF Prevalência de intercorrências relacionadas à amamentação em puérperas. Rev Fac Cienc Med Sorocaba. 2019;20(4):212-7. http:// dx.doi.org/10.23925/1984-4840.2018v20i4a6

39. Batista CLC, Ribeiro VS, Nascimento MDSB, Rodrigues VP. Association between pacifier use and bottle-feeding and unfavorable behaviors during breastfeeding. J Pediatr. 2018;94(6):596-601. http://dx.doi. org/10.1016/j.jped.2017.10.005. PMid:29136496.

40. Mendes SC, Lobo IKV, Sousa SQ, Vianna RPT. Factors associated with a shorter duration of breastfeeding. Cien Saude Colet. 2019 maio;24(5):1821-9. http://dx. doi.org/10.1590/1413-81232018245.13772017. PMid:31166515.

41. Sousa ELO, Melo LGNS, Medeiros DMF. Práticas de complementação ao leite materno : concepções de puérperas sobre aleitamento materno e uso de fórmula infantil. Rev Bras Educ Saúde. 2019;9(2):76-84. http:// dx.doi.org/10.18378/rebes.v9i2.6149.

42. Galindo No NM, Alexandre ACS, Barros LM, Sá GGM, Carvalho KM, Caetano JA. Creation and validation of an educational video for deaf people about cardiopulmonary resuscitation. Rev Lat Am Enfermagem. 2019 mar;27:e3130. http://dx.doi.org/10.1590/1518-8345.2765.3130. PMid:30916231.

43. Franco MS, Carvalho JW, Lira DS, Reis ER, Cirino IP, Lima LHO. Tecnologia educacional para empoderamento materno na autoeficácia educational technology for empowerment in maternal breastfeeding self-efficacy. Rev Enferm UFPE. 2019;13:240857. http://dx.doi.org/10.5205/19818963.2019.240857

44. Moura IH, Silva AFR, Rocha AESH, Lima LHO, Moreira TMM, Silva ARV Construction and validation of educational materials for the prevention of metabolic syndrome in adolescents. Rev Lat Am Enfermagem. 2017 out;25(0):e2934. http://dx.doi.org/10.1590/1518-8345.2024.2934. PMid:29020125.

45. Pinto RH, Vasconcelos EMR, Leal LP, Santos CR, Sousa SM. Construção e validação do instrumento para avaliar o estresse em professores. Rev Enferm UFPE. 2018 set;12(9):2283-92. http://dx.doi.org/10.5205/1981 8963-v12i9a235121p2283-2292-2018.

46. Cordeiro LI, Lopes TO, Lira LEA, Feitoza SMS, Bessa MEP, Pereira MLD et al. Validação de cartilha educativa para prevenção de HIV/ Aids em idosos. Rev Bras Enferm. 2017;70(4):775-82. http://dx.doi. org/10.1590/0034-7167-2017-0145. PMid:28793108.

47. Ximenes MAM, Fontenele NAO, Bastos IB, Macêdo TS, Galindo No NM, Caetano JA et al. Construction and validation of educational booklet content for fall prevention in hospitals. Acta Paul Enferm. 2019 ago;32(4):433-41. http://dx.doi.org/10.1590/1982-0194201900059.

48. Perdigão MMM, Rodrigues AB, Magalhães TL, Freitas FMC, Bravo LG Oliveira PP. Educational technology for fatigue management related to antineoplastic chemotherapy. Rev Bras Enferm. 2019 dez;72(6):151925. http://dx.doi.org/10.1590/0034-7167-2018-0505. PMid:31644739.

49. Santiago JCS, Moreira TMM. Booklet content validation on excess weight for adults with hypertension. Rev Bras Enferm. 2019 fev;72(1):95-101. http://dx.doi.org/10.1590/0034-7167-2018-0105. PMid:30916273.

50. Salbego C, Nietsche EA, Teixeira E, Girardon-Perlini NMO, Wild CF, Ilha S. Care-educational technologies: an emerging concept of the praxis of nurses in a hospital context. Rev Bras Enferm. 2018;71(6, Supl. 6):2666-74. http://dx.doi.org/10.1590/0034-7167-2017-0753. PMid:30540042.

51. Kassianos AP, Ward E, Rojas-Garcia A, Kurti A, Mitchell FC, Nostikasar $D$ et al. A systematic review and meta-analysis of interventions incorporating behaviour change techniques to promote breastfeeding among postpartum women. Health Psychol Rev. 2019;13(3):344-72. http://dx.doi.org/10.1080/17437199.2019.1618724. PMid:31117897.

a Artigo extraído do estudo em andamento "Efetividade de uma intervenção educativa por telefone na promoção do aleitamento materno no período neonatal", coordenado por Maria Augusta Rocha Bezerra. 